\title{
Modern Scale Based Magneto Resistive Sensors Systems
}

\author{
A. Voss, A.Meisenberg, A.Bartos \\ MEAS Deutschland $\mathrm{GmbH}$, a subsidiary of TE Connectivity \\ Hauert 13, 44227 Dortmund \\ Andreas.Voss@te.com
}

\begin{abstract}
Position sensors based on the magneto resistance effect combine high precision with cost effectiveness for linear displacement measurements also in harsh environments where they have clear advantages versus optical encoder systems. Coming from applications with medium accuracy demands but high mechanical tolerances, the whole spectrum of displacement tasks down to nanoscale measurements can be solved with scale based magneto resistive sensor systems. Embedded in a TDFN package for standard, flat or perpendicular mounting, the linear magneto resistive sensors can now easily be applied to a wide range of applications with small design space using standard SMT assembly processes.
\end{abstract}

Key words: magnetoresistive, sensor, scale, linear, package

\section{Magneto resistive sensor systems - what's behind}

Among the variety of technologies for position sensing, solutions based on the magneto resistance (MR) effect [1,2] combine high precision and cost efficiency. As the measurement is contactless, MR based devices work wear free over a wide temperature range in harsh environments [3]. Its components may also be protected against chemicals and dirt, without affecting the precision of the measurement.

In case of linear position measurements, the magnetic field can be provided by a magnetic scale [4], which consists of periodically alternating poles with a base pole length $p_{0}$.

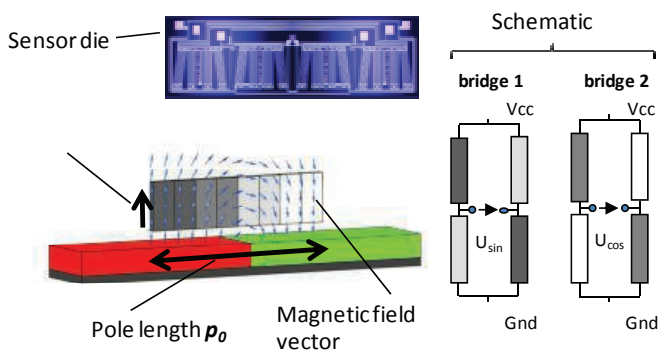

Figure 1 Linear magneto resistive sensor, alignment of Wheatstone bridge

The use of special chip layouts leads to harmonic free sensor output signals, which can also be independent for a wide range of disturbing field strengths. The sensor chips often integrate the magnetic field over more than one pole to enable high accurate measurements. Providing a standard sine and cosine signal, the MR sensors are connected to evaluation ASICs or MCUs. This compact measurement system provides the transformation of an angular magnetic measurement to a digital position information.

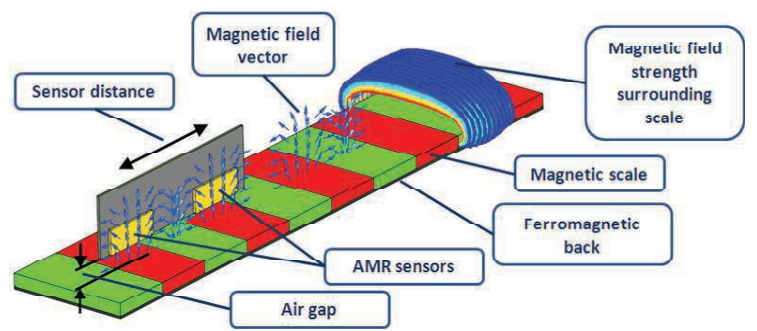

Figure 2 Measurement principle with linear magneto resistive sensors at magnetic scales

\section{AMR signal effects}

Basically an AMR sensor consists of meander formed parallel stripes made of thin NiFe-alloy. The vector between external magnetization and current direction flowing through each meander strip determines the signal component in each meander strip. However in addition to the basic signal there are harmonics with the integral fraction of $180^{\circ}$ by which affect the output signal. 


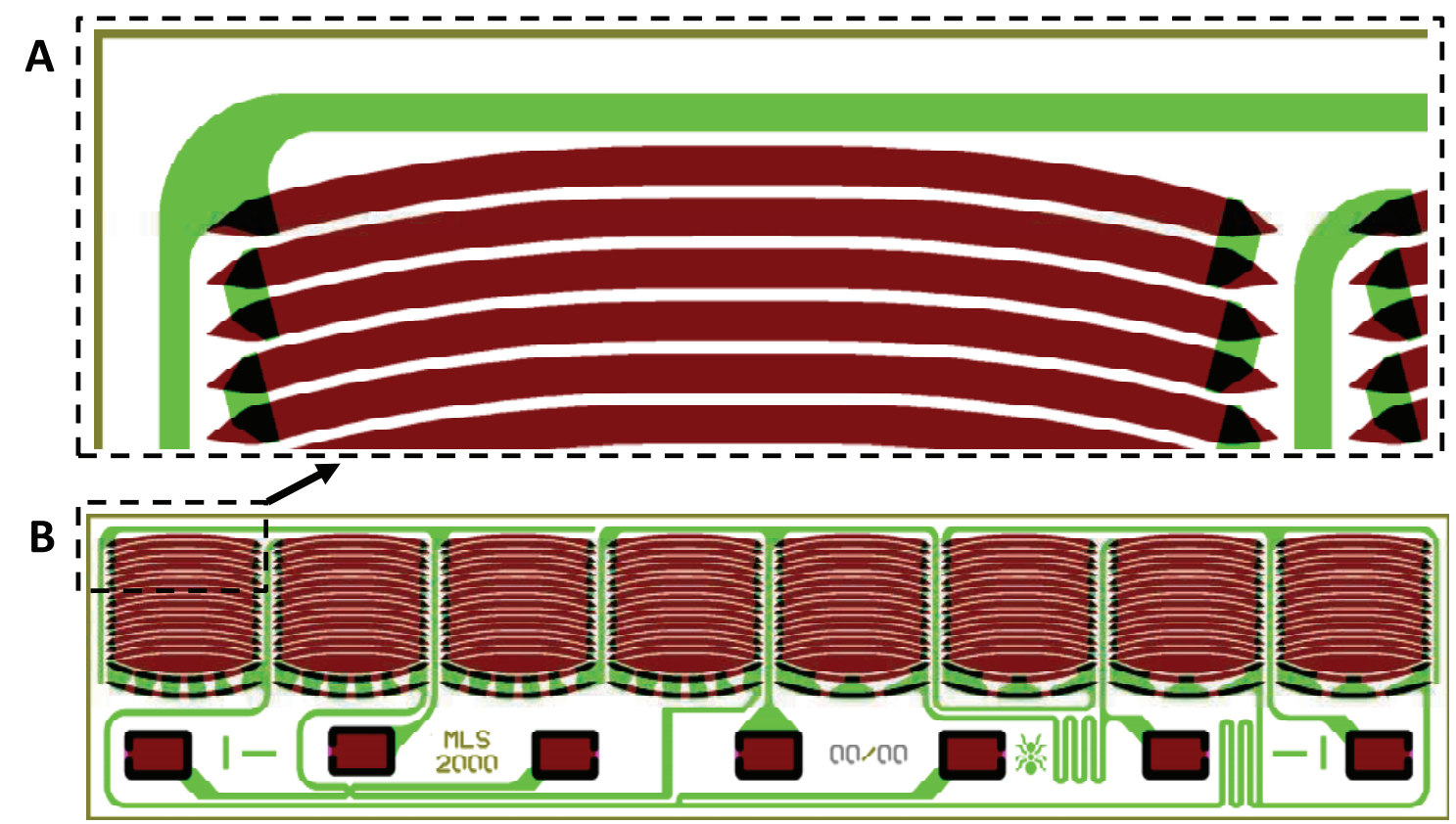

Figure 3 Chip layout of linear AMR sensor, A: parallel curved NiFe-stripes, connected in meander structure B: eight fields of meander stripes lead to two Wheatstone bridges

The assumption of having a corresponding magnetization direction in the meander strip imprinting the external magnetic field direction fails because of two reasons:

1. Anisotropic field acts against external field arising from geometric form and material properties of the meander strip [5].

2. Pinning of meander strip edge domains.

As a result, the signal contains a deviation of the expected ideal change of resistance and hysteresis behavior [6].

Using stronger magnetic fields will decrease both effects by forcing the alignment of the domains to the external field direction. A more suitable way of compensating harmonics even at lower fields is done by consciously created inverted harmonic sub-signals, which will interfere destructively. This is enhanced by the meander strip layout.

\section{Harmonic compensation by shape of meander stripe}

Using a linear AMR sensor which is placed perpendicular to a scale needs filtering of harmonics in the sensor signal by splitting straight parts of the meander strips into two or more groups of defined angles between the parallel directions of the meander strips.

Increasing the number of groups leads to a higher order of harmonics, which will be filtered out of the sensor signal. As a disadvantage the separation of the strip into sub-stripes may lead to pinning effects on the edges between each group.
Using a curved shape for the meander stripes, leads to a continuous and simultaneous propagation of the domains. This eliminates the domain pinning effects. Finally an isotropic layout approach leads to a symmetric pattern of meander areas where no field direction is preferred. The corresponding sensor layout show a high gain in accuracy compared to the simple flat stripe design. This is shown in figure 4.
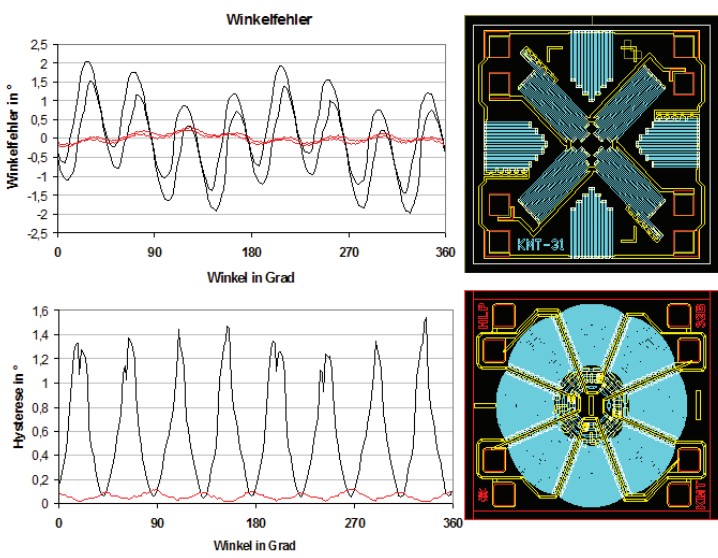

Figure 4 Performance comparison between flat and curved meander stripe design. Red: accuracy, black: hysteresis, measured at applied field strength of 8 $k A / m$

\section{Example for harmonic compensation by geometrical shift of meander stripes}

Looking to a sin/cos position sensor placed in plane on top of a magnetic scale the following way of harmonic compensation is typically used. The periodicity is $2 \mathrm{~mm}(1 \mathrm{~mm}$ north pole, $1 \mathrm{~mm}$ south pole), basic frequency $=\mathrm{f} 1$. 

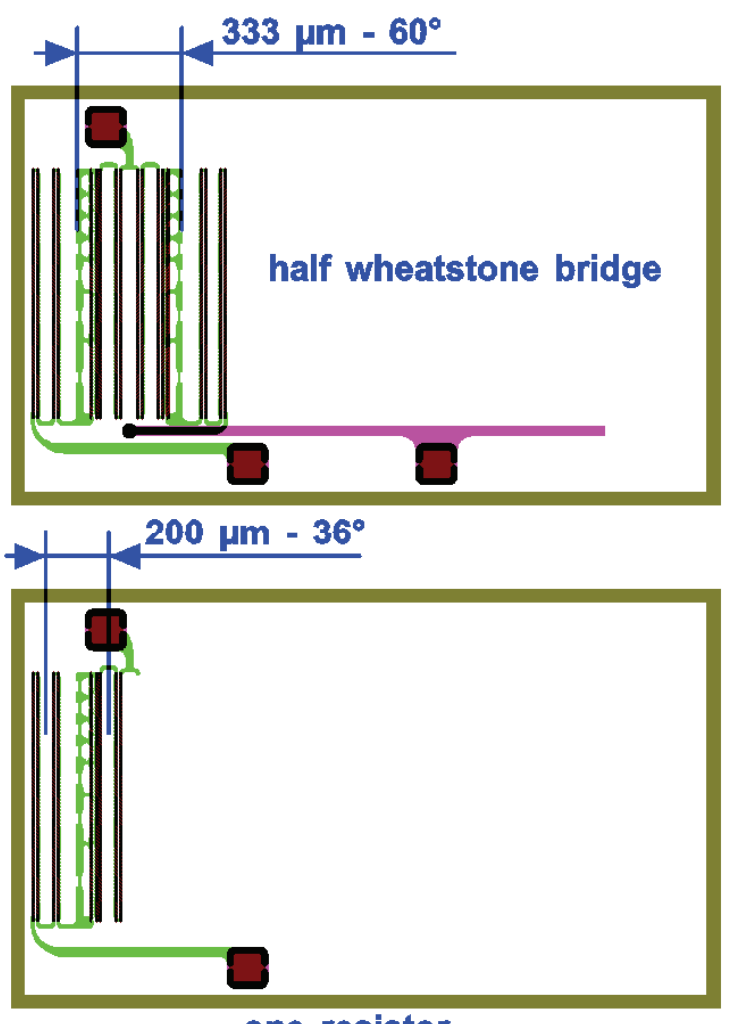

one resistor sin- and cos-wheatstone bridge
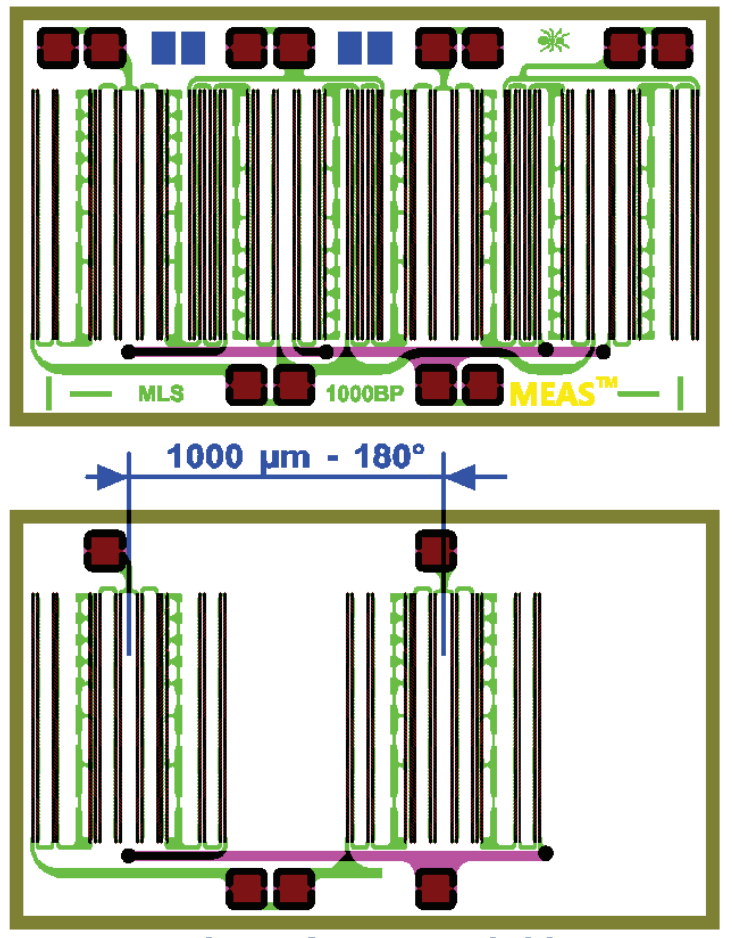

complete wheatstone bridge

Figure 5 Chip layout of linear AMR sensor for in plane applications, harmonic compensation through geometrical displacement of compensation fields

One resistor contains two meander groups with a distance of $200 \mu \mathrm{m}$ or $36^{\circ}$ phase difference. This group eliminates the 5th harmonic. The resistors form a half Wheatstonebridge, having a distance of $333 \mu \mathrm{m}$ or $60^{\circ}$ phase difference. This combination eliminates the 3th harmonic. Both half bridges are combined to a Wheatstone bridge, which have a distance of $1000 \mu \mathrm{m}$ or $180^{\circ}$ phase difference. This combination eliminates all even harmonics and so forth.

\section{Modern DFN packages allow new applications}

The integration of the sensor head and the scale into a mechanical system is very often restricted by space limitations.

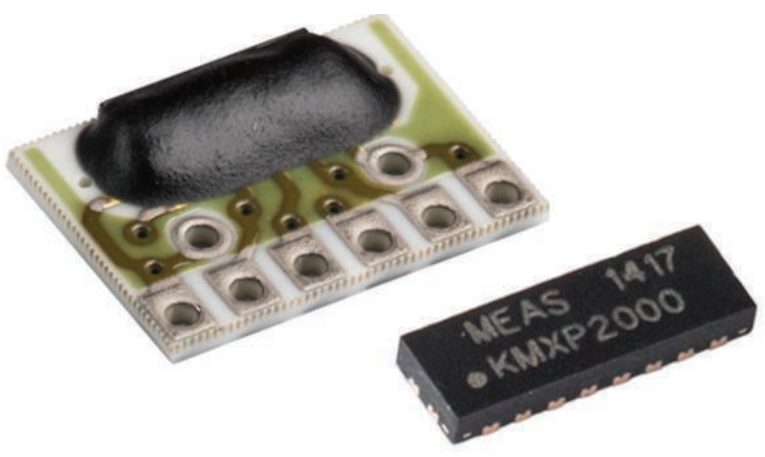

Figure 6 Comparison between COB based chip assembly on ceramic and DFN package
Here the use of modern DFN packages in combination with new scale materials, that allow much thinner scale thickness, opens the door for new applications, where precise linear displacement measurements are needed in a very limited space.

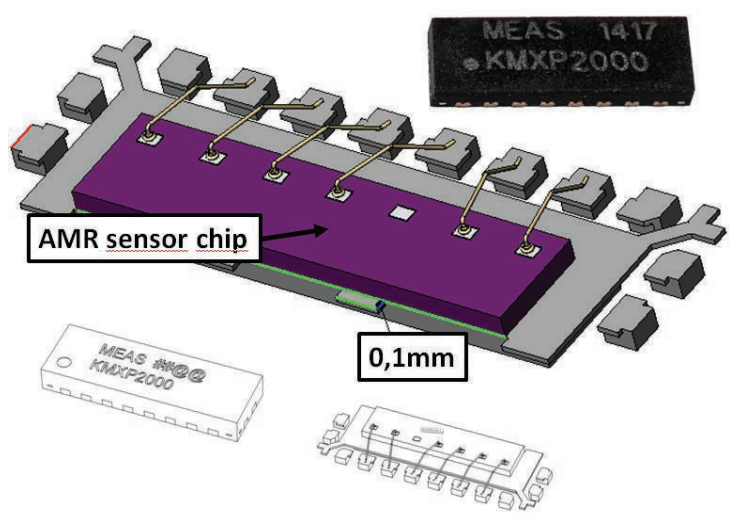

Figure 7 Assembly of KMXP series DFN packaged $A M R$ linear sensor, maximum air gap of sensor to package edge $=0,1 \mathrm{~mm}$

As the sensor chip has to be placed as close as possible to the scale a typical assembly technology today is chip-on-board (COB, see figure 6)), which is a costly, non-standard assembly process. Modern DFN packages are tiny, robust and most importantly, allow standard 
SMT processes. They provide much better defined mechanical tolerances as standard glob tops (see figure 7), with much less mechanical internal stress by giving a robust protection to the sensor chip and a well defined placement on the printed circuit board.

\section{Perpendicular and flat sensor to scale alignments}

The following two examples illustrate where the beneficial properties of DFN packages apply: Figure 8 shows the common application, where the sensor is placed on the edge of the PCB. DFN packages also allow a perpendicular assembly, so that the PCB can now be placed parallel to the scale. The sensor chip itself stays at perpendicular alignment to the scale.

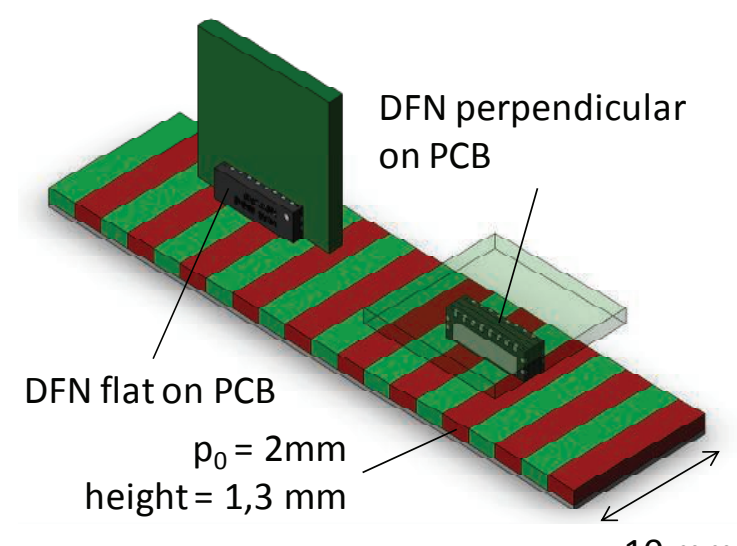

Figure 8 flat and perpendicular DFN packaged AMR sensor, air gap: 1mm @ $p_{0}=2 \mathrm{~mm}$, chip alignment to scale remains perpendicular

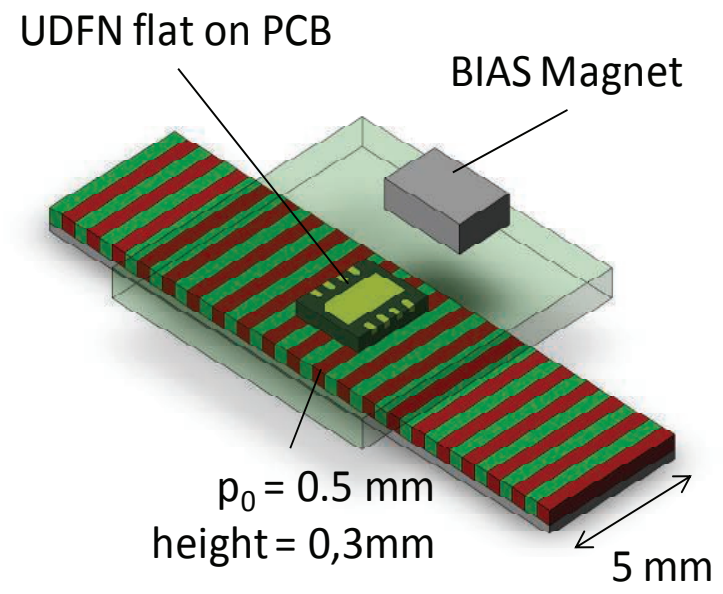

Figure 9 extra thin track (height $=0,3 \mathrm{~mm}$ ) with flat UDFN packaged MR sensor, air gap:0,125mm @ p $p_{0}=$ $0,5 \mathrm{~mm}$, flat chip alignment to scale leads to very small air gaps
For more precise measurements very small pole pitches are needed which in turn requires very small air gaps. Figure 9 depicts how this measurement task can be addressed utilizing an ultrathin DFN package.

\section{Linear measurement systems based on DFN packaged linear AMR sensors}

When designing a linear magnetic encoder one has to take 3 parameters into account:
1. Used sensor principle
2. Magnetic scale
3. Application

The overall system accuracy depends mainly on a small tolerance band of the air gap between sensor and scale as well as of the scale quality itself.

Common scale qualities ensure accuracies of $+/$ $40 \mu \mathrm{m} / \mathrm{m}$ down to $+/ 10 \mu \mathrm{m} / \mathrm{m}$ [7]. This accuracy field is often reached after just some millimeter scale although it is not exceeded over 1 meter scale as defined.

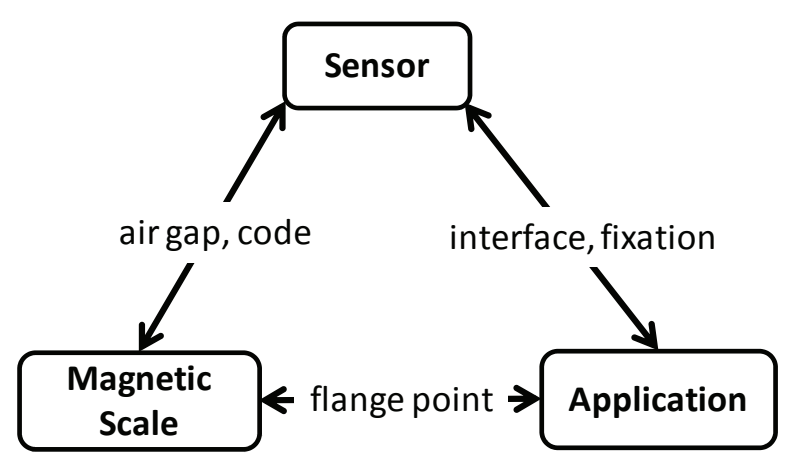

Figure 10 Triangle of Encoder design, dependencies of design parameters

Using DFN packaged linear AMR sensors enable a maximum air gap between scale and sensor as the component could be placed directly to the edge of the PCBA. The three-sided pad layout ensures exact alignment during the SMT soldering process.

As result high accurate linear encoders down to +/- $3 \mu \mathrm{m}$ accuracy could be generated when combined with a high accurate magnetic scale as shown in figure 11. 


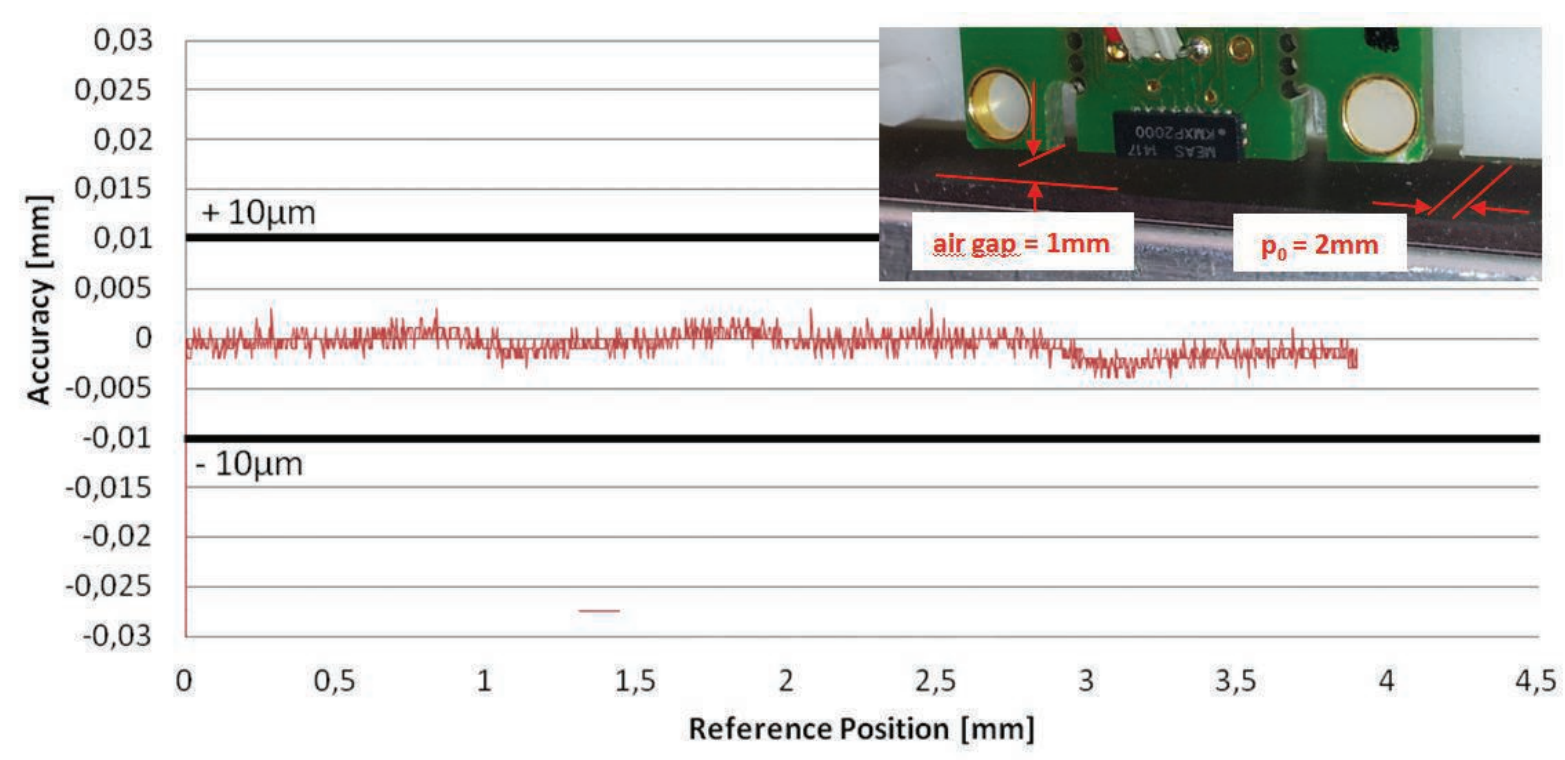

Figure 11 Accuracy plot of a flat mounted KMXP2000 DFN magnetic length sensor, using pole pitch $p_{0}=2 \mathrm{~mm}$

\section{References}

[1] U. Dibbern: Magnetic field sensors using the magnetoresistive Effect, Sensors and Avtuators, 10, pp 127-140,1986

[2] T.R. McGuire, R.T. Potter: Anisotropic magnetoresistance in ferromagnetic $3 d$ alloys, IEEE Transactions on Magnetics, Vol. Mag-11, No- 4, 7/1975

[3] T. Reiniger, C. Harnisher: Magnetic field sensors for the industrial automation. Sensors and Actuators, A59 , pp 177 - 182, 1997

[4] A.Voss, A.Meisenberg, R.Pieper, A.Bartos: Absolute Positionsbestimmung mit magnetoresistiven Sensoren , Mikrosystemtechnik Kongress 2013 pp 215 218, ISBN 978-3-8007-3555-6

[5] S.Shtrikman, D.R. Smith: Analytical formulas for the unshielded magnetoresistive head, IEEE TRANSACTIONS ON MAGNETICS. VOL 32, NO 3. MAY 1996

[6] A.Bartos: Magnetische Messtechnik am Beispiel der AMR Messtechnik, TAE Seminar Magnetische Messtechnik - Geräte, Verfahren, Anwendungen, Esslingen 21.11.207

[7] Messprotokoll „Lineare magnetische Maßstäbe“ BOGEN Electric GmbH 25.07.2012 